\title{
Sutura intercrura lateral no nariz caucasiano: Estruturação e diminuição do ângulo de divergência domal na rinoplastia endonasal sem delivery
}

\author{
Lateral intercrural suture in the caucasian nose: Decreased domal divergence angle in \\ endonasal rhinoplasty without delivery
}

\author{
Cezar Augusto Sarraf Berger ${ }^{1}$, Marcos Mocelin², Caio Márcio Correia Soares ${ }^{3}$, Rogério Pasinato ${ }^{4}$, Andreia Ellery Frota ${ }^{5}$. \\ 1) Mestre em Clínica Cirúrgica. Professor voluntário do Departamento de Otorrinolaringologia da Universidade Federal do Paraná. Médico Otorrinolaringologista do Hospital \\ IPO. \\ 2) Professor Titular do Departamento de Otorrinolaringologia da Universidade Federal do Paraná(UFPR). Médico Otorrinolaringologista do Hospital IPO. \\ 3) Mestre em Clínica Cirurgica. Médico Otorrinolaringologista do Departamento de Otorrinolaringologia da Universidade Federal do Paraná (UFPR) e do Hospital IPO. \\ 4) Mestre. Professor Adjunto do Departamento de Otorrinolaringologia da Universidade Federal do Paraná (UFPR). Médico Otorrinolaringologista do Hospital IPO. \\ 5) Especialista em Otorrinolaringologia. Médica Otorrinolaringologista. Ex-Fellow de Cirúrgia Plástica da Face do Hospital IPO
}

Instituição: Instituto Paranaense de Otorrinolaringologia

Curitiba / PR - Brasil.

Endereço para correspondência: Cezar Augusto Sarraff Berger - Avenida República Argentina 2069 - Bairro Água Verde - Curitiba / PR - Brasil - CEP: 80620 -010 - Telefone: (+55 41) 3314-1503 - E-mail: nep@ipo.com.br

Artigo recebido em 14 de outubro de 2011. Artigo aprovado em 21 de fevereiro de 2012.

\section{RESUMO}

Introdução: Diversas técnicas podem ser realizadas para melhorar a definição da ponta nasal como ressecção cartilaginosa, colocação de enxertos ou suturas. A realização de suturas na ponta nasal proporciona resultados estéticos satisfatórios com menor morbidade1-5.

Objetivo: Avaliar a sutura intercrura lateral realizada na cartilagem lateral inferior, através de rinosseptoplastia endonasal por técnica básica sem delivery, para diminuição do ângulo de divergência domal no nariz caucasiano e consequente melhora na definição da ponta nasal.

Método: Realizado estudo prospectivo com 64 casos nos quais foi confeccionada sutura no bordo cefálico da cartilagem lateral inferior na junção entre a cúpula e crus lateral, utilizando-se fio P.D.S. (Polydioxanorie ${ }^{\circledR}$ ) incolor 4 "0" com agulha curva cortante. Resultado: Foram analisadas e comparadas as fotos do pré - operatório e do pós -operatório com 6 meses de evolução. Em todos os casos foi atingida uma melhora na definição da ponta através da sutura intercrura lateral.

Conclusão: A sutura intercrura lateral da cartilagem lateral inferior mostrou ser factível para uma melhor definição da ponta no nariz caucasiano podendo ser realizada por rinoplastia endonasal sem delivery.

Palavras-chave: suturas, rinoplastia, cartilagens nasais.

\section{SUMMARY}

Introduction: Several techniques can be performed to improve nasal tip definition such as cartilage resection, tip grafts, or sutures.

Objctive: To evaluate the outcome of lateral intercrural suture at the lower lateral cartilage by endonasal rhinoplasty with a basic technique without delivery in decreasing the angle of domal divergence and improving the nasal tip definition.

Method: This prospective study was performed in 64 patients in which a suture was made on the board head of the lower lateral cartilage in the joint between the dome and lateral crus, using polydioxanone (PDS) with sharp, curved needle.

Results: In all of the cases, better definition of the nasal tip was achieved by intercrural suturing for at least 6 months postoperatively.

Conclusion: Lateral intercrural suture of the lower lateral cartilage provides improved nasal tip definition and can be performed by endonasal rhinoplasty without delivery in the Caucasian nose.

Keywords: sutures, rhinoplasty, nasal cartilage. 


\section{INTRODUÇÃO}

A cirurgia da ponta nasal representa um desafio na rinosseptoplastia. Diversas técnicas podem ser realizadas para melhorar a definição da ponta nasal como ressecção cartilaginosa, colocação de enxertos ou suturas. Ao longo dos anos, técnicas de ressecção cartilaginosa têm sido menos utilizadas, havendo aumento da indicação de técnicas que preservem mais os mecanismos de sustentação da ponta nasal (1-10).

O objetivo deste estudo é descrever a sutura intercrura lateral simples realizada na cartilagem lateral inferior do nariz caucasiano, através de rinosseptoplastia endonasal por técnica básica sem delivery, para diminuição do ângulo de divergência domal e melhor definição da ponta.

\section{MÉTODO}

O estudo foi realizado nos anos de 2008 e 2009 no Hospital Paranaense de Otorrinolaringologia (IPO) em Curitiba-Parana. De um total de 100 pacientes submetidos a rinosseptoplastia de forma consecutiva, foram excluídos os pacientes com história de cirurgia nasal, trauma nasal pregresso e narizes não caucasianos. Participaram do estudo 64 pacientes, masculinos $=43$, femininos $=21$, com idade variando de $16-59$ anos. ( $\mathrm{n}=37,5)$. Todos os pacientes tinham conhecimento do estudo e assinaram o TCLE conforme a resolução CNS 196/96. A técnica cirúrgica aplicada foi a rinosseptoplastia clássica de ConverseDiamond (1). O acesso a ponta nasal foi realizado através da incisão septocolumelar e intercartilaginosa. Foi realizada a manobra de La Garde em todos os casos sem ressecção da borda cefálica da cartilagem lateral inferior (CLI) (técnica de McIndoe).

A sutura intercrura lateral deve ser realizada no bordo cefálico da CLI através da incisão intercartilaginosa, na junção entre a cúpula e a crus lateral (Figura 1). Seu aperto deve ser gradual e progressivo, permitindo ajustes quanto à definição da ponta mais adequada (Figura 2) .

A sutura intercrura lateral deve ser realizada após a sutura septocolumelar e antes da sutura intercartilaginosa, utilizando-se o fio PDS ${ }^{\circledast}$ incolor 4"0" com agulha curva cortante. A realização do ponto intercrural no final da cirurgia evita manipulação excessiva da ponta e possível alargamento da distância interdomal (ângulo de divergência domal).

Considera-se normal um ângulo de divergência domal menor que $30^{\circ}$ (Figura 3).

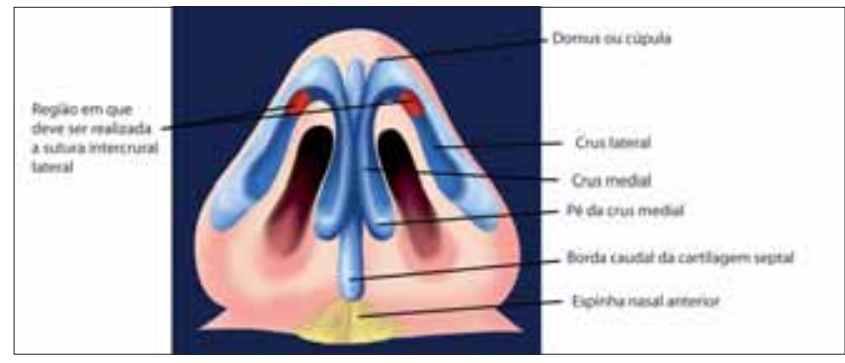

Figura 1. Anatomia cirúrgica da ponta nasal.
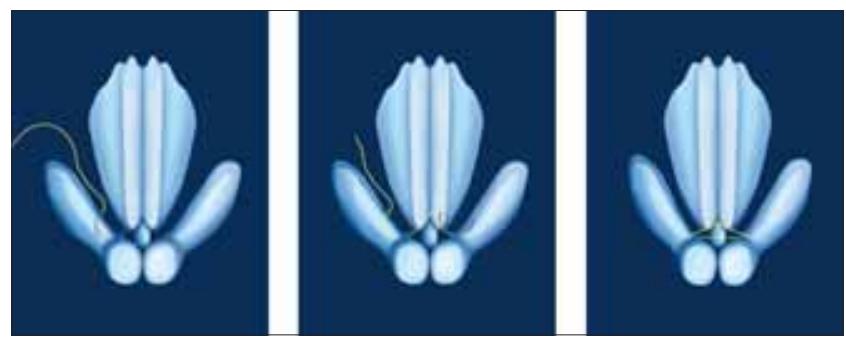

Figura 2. Confecção da sutura intercrura lateral.

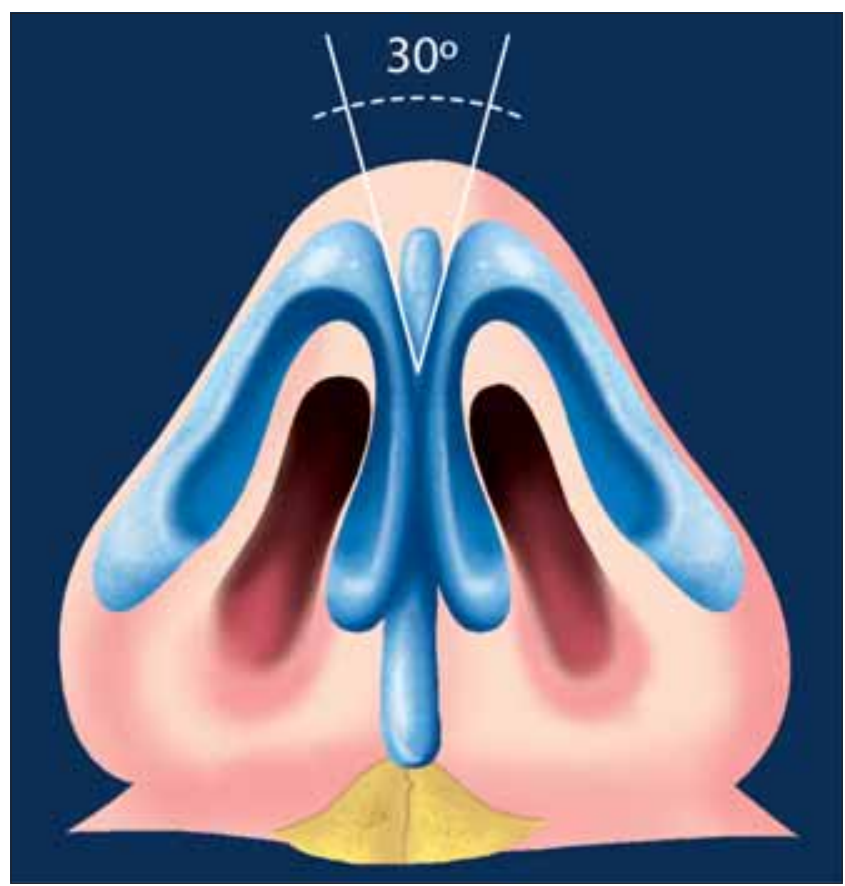

Figura 3. Ângulo de divergência domal normal.

Passos para confecção da Sutura Intercrura lateral (Descrição da técnica):

1. Após descolamento da CLI da pele através da manobra de La Garde e descolamento da cartilagem da pele do vestíbulo, transpassar o fio P.D.S. ${ }^{\oplus}$ na junção entre a cúpula e a crus lateral (cerca de 3 mm do bordo cefálico da CLI). O fio deve ser passado em sentido céfalocaudal (Figura 4; Passo 1). 
2. Transpassar o fio para a narina contralateral através da incisão transfixante (Figura 5; Passo 2).

3. Realizar o passo 1 na narina contralateral, em sentido caudo-cefálico, evitando-se assim o cruzamento do fio (Figura 6; Passo 3).

4. Retornar a agulha para a narina em que se iniciou o ponto, realizando o aperto da sutura de modo gradual.

\section{RESULTADOS}

A Sutura Intercrura Lateral foi realizada em 64 pacientes com nariz caucasiano através da rinosseptoplastia endonasal por técnica básica sem delivery. Foram avaliadas as fotos do pré e pós- operatório com 6 meses de evolução. Em todos os casos foi atingida uma melhor definição da ponta nasal com resultados satisfatórios para o paciente e o cirurgião (avaliação qualitativa, observacional e subjetiva). Não houve nenhum caso de complicação.

Este trabalho foi aprovado pela comissão de ética da instituição (protocolo número 004/2008).

\section{DISCUSSÃO}

A realização de suturas para melhora da definição da ponta nasal é uma tendência mundial (5), em detrimento das técnicas de ressecção cartilaginosa. As principais suturas usadas na ponta nasal foram publicadas por BAHMAN Guyuron e Ramin A. Behmand em 2003 (6).

Nossos resultados foram avaliados com 6 meses de pós operatório e com sucesso semelhante ao trabalho publicado por Nassif FilHo e colaboradores (11).

O resultado final da sutura é influenciado principalmente por fatores como: a força intrínseca da cartilagem; o grau de aperto do ponto; a limitação imposta pelos tecidos moles (ligamentos, tecido subcutâneo e pele).

A espessura da pele é um fator determinante na eficácia da Sutura Intercrura Lateral. Em pacientes com pele fina e tecido subcutâneo escasso os resultados são mais expressivos. Já em pacientes com pele muito grossa e excesso de tecido subcutâneo, a realização da Sutura Intercrural pode se tornar pouco efetiva, devendo-se realizar a diminuição do ângulo de divergência domal e de definição domal através de outras técnicas como a Técnica de Goldman, a sutura transdomal, interdomal, "medial crus suture"(rotação / projeção) ou a sutura tipo "alarlateral spanning" (comprometendo mais a crus lateral) (6).

A sutura intercrura lateral possui detalhes os quais devem ser observados:

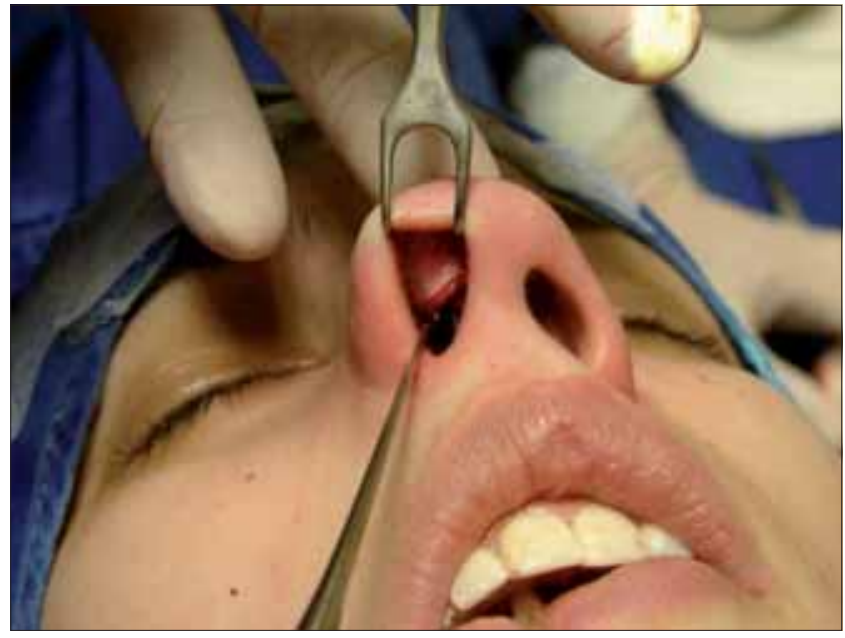

Figura 4. Passo 1.

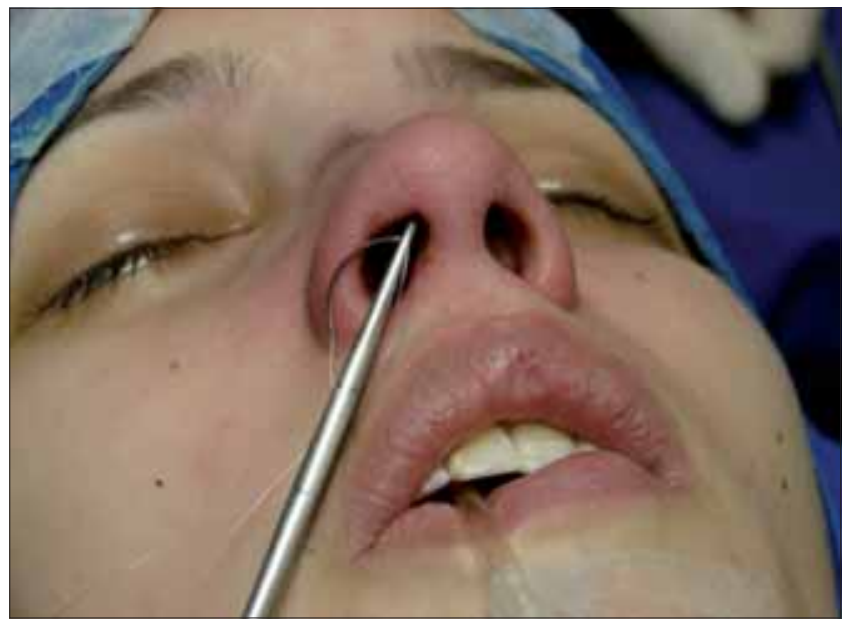

Figura 5. Passo 2.

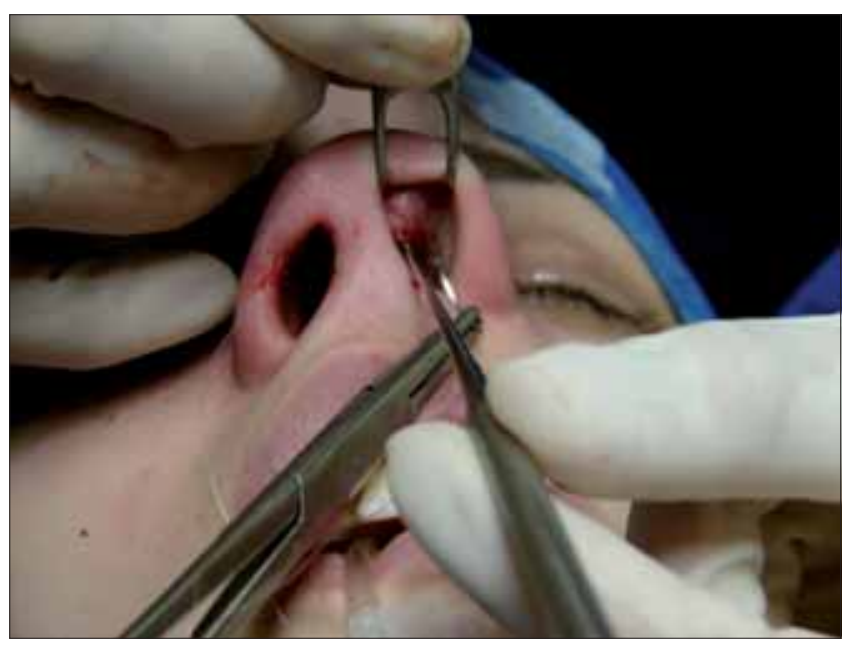

Figura 6. Passo 3. 
1. Leve projeção da ponta, divergindo do descrito na literatura (2). O grau de definição da ponta depende da localização em que é realizado o ponto. Quanto mais lateral na cruz lateral é realizada a sutura, maior a definição e a projeção da ponta nasal.

2. Discreto pinçamento da região supra-alar, principalmente em pacientes com pele muito fina e cartilagem lateral débil, quando realizada em distância maior de 2$3 \mathrm{~mm}$ da cúpula nasal.

3. Assimetria de ponta se o ponto não for realizado em níveis equivalentes.

4. Pollybeaksecundário.

A sutura pode ser realizada com fios absorvíveis de longa duração (monocryl, PDS) ou com fios inabsorvíveis, considerando-se a força tênsil e o grau de reabsorção de cada fio. Em todos nossos casos foi utilizado fio inabsorvível não havendo nenhum caso de extrusão ou outras complicações descritas na literatura (abscesso, extrusão do fio, assimetria da ponta, pinçamento da ponta) (2).

\section{CONCLUSÃO}

A sutura intercrura lateral é um método efetivo para melhor definição da ponta nasal no nariz caucasiano podendo ser realizado através da rinosseptoplastia endonasal sem delivery. Tal técnica já havia sido descrita na literatura através dos acessos aberto e fechado via delivery, mas não através de acesso fechado com incisões intercartilaginosa e septocolumelar.

É uma sutura de fácil realização, previsível, controlada e extremamente útil no arsenal do cirurgião para a cirurgia da ponta. Este ponto permite o melhor manejo da ponta através da rinosseptoplastia por técnica básica, resultando em uma diminuição da necessidade de realização de cirurgia por via delivery ou via aberta.

O presente estudo avaliou a melhora da definição da ponta na região do ângulo de divergência domal com a sutura simples do bordo cefálico da crus lateral da CLI. Destacamos que cada sutura tem sua indicação precisa, cabe ao cirurgião julgar e optar pela melhor técnica.

\section{REFERÊNCIAS BIBLIOGRÁFICAS}

1. Maniglia AJ, Maniglia JJ, Maniglia JV. Rinoplastia EstéticaFuncional-Reconstrutora. 1를 ed. Revinter. 2002, 129-150.

2. Corrado A, Bloom J, Becker D. Domal Stabilization Suture in Tip Rhinoplasty. Arch Facial Plast Surg. 2009, 11(3):1947.

3. Tardy ME, Brown RJ. Surgical anatomy of the nose. New York: Raven Press, 1990.

4. Rohrich RJ, Adams WP. The Boxy Nasal Tip: Classification and Management Based on Alar Cartilage Suturing Techniques. Plast Reconst Surg. 2001, 1849:107

5. Tebbetts JB. Discussion:nasal tip sutures part I: the evolution. Plast Reconst Surg. 2003, 12(8):1146-9.

6. Guyuron B, Behmand R. Nasal tip sutures part II: the interplays. Plast Reconst Surg. 2003, 12(8):1146-9.

7. LoS, Rowe-Jones J. Suture techniques in nasal tip sculpture: current concepts. J Laryngol Otol. 2007, 121(8):e10.

8. Gruber R, WeintraubJ, pomerantzJ. Sutura Techniques for Nasal Tip. Aesthetic Surg J. 2008, 28:92-100.

9. Perkins S, Patel A. Endonasal Suture Techniques in Tip Rhinoplasty. Facial plast Surg Clin North Am. 2009, 17(1):4154.

10. Leach JL, Athré. Four suture tip rhinoplasty: A powerful tool for controlling tip dynamics. Otol Head Neck Surg. 2006, 135:227-31.

11. Nassif Filho ACN, Romano G, Ribas DB, Sass SMG, Franceschi E. Refinamento da ponta nasal: técnica de sutura minimamente invasiva para a ponta nasal larga. Arq. Int. Otorrinolaringol. 2011, 15(3):302-307. 